\title{
Intractable paroxysmal tachycardia caused by a concealed retrogradely conducting Kent bundle Demonstration by epicardial mapping and cure of tachycardias by surgical interruption of the His bundle ${ }^{1}$
}

\author{
Pablo Denes, Christopher R. C. Wyndham, and Kenneth M. Rosen \\ From th: Cardiology Section, Dzpartment of Medicine of the Abraham Lincoln School of Medicine, University \\ of Illinois College of Medicine, Chicago, Illinois, U.S.A.
}

Electrophysiological and epicardial mapping studies are described in a patient without pre-excitation who had intractable recurrent paroxysmal suprazentricular tachycardia. Electrophysiological studies revealed fixed $V A$ conduction times during both rapid ventricular pacing and coupled ventricular stimulation. Catheter mapping of atrial activation during retrograde conduction and during induced paroxysmal supraventricular tachycardia revealed early distal ccronsry sinus activation (posterior left atrium) relative to the low septal, low lateral, and high lateral right atrium. These studies suggested the presence of a concealed left-sided bypass tract.

The patient underwent surgical interruption of the His bundle for control of paroxysmal supraventricular tachycardia. Epicardial mapping of the atria (during ventricular pacing) confirmed the presence of a concealed left-sided bypass tract. Surgery produced antegrade $A V$ block (while retrograde conduction was maintained) and total cure of paroxysmal supraventricular txchycardia. This is the first reported case of a concealed retrograde extranodal pathway documented by epicardial mapping.

Recent studies in patients with re-entrant paroxysmal supraventricular tachycardia without apparent pre-excitation suggest that re-entry can either be within the AV node or involve the normal pathway and a concealed extranodal pathway (Denes et al., 1973; Coumel and Attuel, 1974; Neuss, Schlepper, and Thormann, 1975; Spurrell, Krikler, and Sowton, 1974a, b; Zipes, DeJoseph, and Rothbaum, 1974). In the latter cases, the extranodal pathway is capable of only retrograde conduction and, therefore, concealed on the surface electrocardiogram. Differentiation between intra- and extranodal reentry has both diagnostic and therapeutic implications.

In the present report, we describe the electrophysiological and epicardial mapping studies in a

1Supported in part by a United States Public Health Service training grant. patient without ventricular pre-excitation who had recurrent, intractable supraventricular tachycardia, and who underwent surgical ablation of the His bundle. Preoperative electrophysiological studies suggested the presence of a left-sided concealed extranodal pathway capable of only retrograde conduction. Epicardial mapping of the atria at the time of cardiac surgery confirmed the presence of an anomalous left-sided connexion. After surgical section of the His bundle, complete antegrade AV block was produced with maintenance of intact retrograde conduction.

\section{Case report}

The patient was a 44-year-old male Jehovah's witness, with documented recurrent paroxysmal supraventricular tachycardia for 15 years. Electrocardiograms between attacks were within normal limits, with a PR interval 


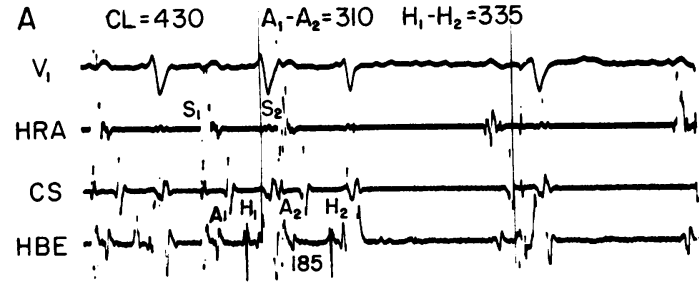

B
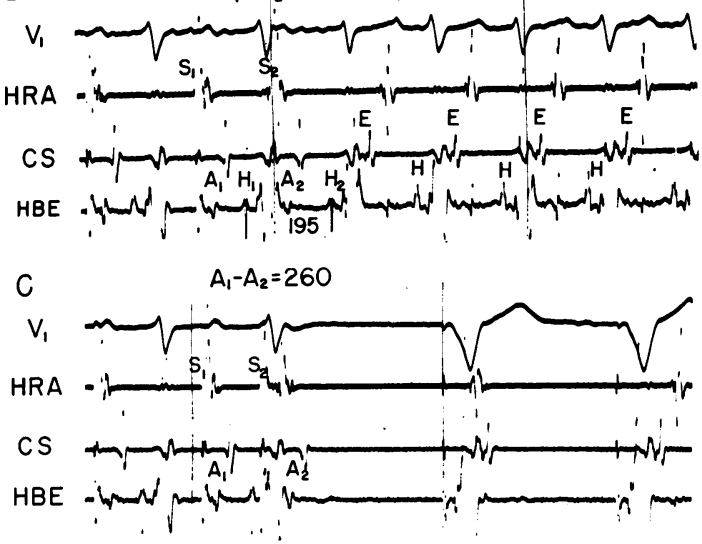

FIG. 1 Induction of paroxysmal supraventricular tachycardia during coupled right atrial pacing at a basic cycle length of $430 \mathrm{~ms}$. In panel $A$, the $A_{1}-A_{2}$ coupling interval is $310 \mathrm{~ms} . A_{2}$ is conducted to the ventricles with an $A H$ interval of $185 \mathrm{~ms}$. In panel $B$, the $A_{1}-A_{2}$ interval has been decreased by $5 \mathrm{~ms}$. $A_{2}-H_{2}$ interval is $195 \mathrm{~ms}$ and $A_{2}$ is followed by an echo beat $(E)$ and an episode of paroxysmal supraventricular tachycardia is induced. Note that the coronary sinus (CS) electrogram of $E$ precedes both high right atrial and low septal right atrial electrograms. In panel $C, A_{1}-A_{2}$ is $260 \mathrm{~ms} . A_{2}$ is blocked in the $A V$ node (proximal to $H$ ). The permanent ventricular pacemaker escapes following the blocked $A_{2}$.

of $0.18 \mathrm{~s}$, and a QRS duration of $0.08 \mathrm{~s}$. Episodes of paroxysmal supraventricular tachycardia were characterized by rates of 170 to 190 /minute and narrow QRS complexes identical to those noted during sinus rhythm.

During the two years before admission, attacks of paroxysmal supraventricular tachycardia had become more frequent and prolonged, occurring several times daily and often lasting for hours. The patient had to give up gainful employment as a lecturer because of the severity and frequency of the attacks. Therapy had included the following in high dosage, both singly and in combination: digitalis, propranolol, quinidine, phenytoin, and procainamide. The patient manifested refractoriness and/or major side effects to all of these drugs.
Because of failure of drug therapy, an attempt was made to control the tachycardia with atrial pacing, but was unsuccessful. A permanent externally activated demand epicardial ventricular pacemaker was implanted, when studies suggested that this mode of therapy would result in interruption of tachycardias. However, within several weeks of pacemaker implantation, intractable recurrent paroxysmal supraventricular tachycardia again occurred. The patient was then referred to the University of Illinois Hospital for further evaluation.

\section{Electrophysiological studies}

Electrophysiological studies were performed in January 1975, after informed consent was obtained. Medicines were discontinued 72 hours before study. His bundle electrograms were recorded with catheter techniques (Scherlag et al., 1969). In addition, a quadripolar catheter was positioned in the high right atrium for recording and stimulation. A hexapolar catheter was positioned so that the distal two poles were located at the right ventricular apex, while the proximal electrodes were contiguous to the low lateral right atrium. A bipolar catheter was placed in the distal coronary sinus.

Basic conduction intervals were recorded during sinus rhythm at a cycle length of $800 \mathrm{~ms}$ and were as follows: $\mathrm{PA}$ of $42 \mathrm{~ms}$, AH of $84 \mathrm{~ms}$, and HV of $45 \mathrm{~ms}$. With high right atrial pacing, 1:1 AV conduction was noted up to a paced rate of $200 / \mathrm{min}$, with an increase in $\mathrm{AH}$ interval from 110 to $200 \mathrm{~ms}$. AV nodal Wenckebach periods were noted at a paced rate of $210 / \mathrm{min}$. There was no evidence of an antegradely conducting anomalous pathway with pacing of the low lateral right atrium, the coronary sinus, and low septal right atrium (Denes et al., 1974). With right ventricular pacing at rates of 90 to $170 /$ min VA conduction (ventricle to low septal right atrium) remained constant at $230 \mathrm{~ms}$. The sequence of atrial depolarization during retrograde conduction was distal coronary sinus, preceding both low septal right atrium and high right atrium by $40 \mathrm{~ms}$.

Antegrade refractory periods were measured with atrial extra-stimulus technique from the high right atrium at cycle lengths of 600 and $430 \mathrm{~ms}$ and are listed in the Table. The AV conduction curves $\left(\mathrm{A}_{1}-\mathrm{A}_{2}, \mathrm{H}_{1}-\mathrm{H}_{2}\right)$ were smooth.

TABLE Refractory periods (ms)

\begin{tabular}{lcc}
\hline$C L$ & 600 & 430 \\
\hline Atrial ERP & 220 & 230 \\
Atrial FRP & 270 & 250 \\
AV node ERP & 270 & 260 \\
AV node FRP & 380 & 330 \\
Ventricular ERP & - & 280 \\
Bypass ERP & & $<280$ \\
AV echo zone & & $305-270$ \\
\hline
\end{tabular}

$\mathrm{CL}=$ Cycle Length; FRP = Functional Refractory Period; $\mathrm{ERP}=$ Effective Refractory Period. 
Retrograde refractory period determinations were performed using the ventricular extrastimulus technique $\left(\mathrm{V}_{2}\right)$ from the right ventricular apex at a cycle length of $600 \mathrm{~ms}$. $\mathrm{V}_{2}-\mathrm{A}_{2}$ remained constant at $230 \mathrm{~ms}$, despite shortening of $V_{1}-V_{2}$ from 580 to $285 \mathrm{~ms}$. The sequence of atrial activation was identical to that described above during incremental ventricular pacing. VA conduction was limited by ventricular refractoriness at an $S_{1}-S_{2}$ interval of $280 \mathrm{~ms}$.

Both single atrial echoes and sustained paroxysmal supraventricular tachycardia were noted during pacinginduced AV nodal Wenckebach periods, with achievement of a critical AH of $260 \mathrm{~ms}$ (Goldreyer and Damato, 1971). An echo zone (single echoes \pm episodes of sustained paroxysmal supraventricular tachycardia) was defined with atrial extrastimulus technique between $A_{1}-A_{2}$ coupling interval of 305 to $270 \mathrm{~ms}$ at a cycle length of $430 \mathrm{~ms}$ (Fig. 1). The critical $\mathrm{AH}$ for induction of echoes during coupled stimulation was $195 \mathrm{~ms}$ (Fig. 1B) (Goldreyer and Damato, 1971).

Induced sustained paroxysmal supraventricular tachycardia was characterized by a narrow QRS, an HV of $45 \mathrm{~ms}$, and a cycle length of $367 \mathrm{~ms}$. Retrograde activation times during induced paroxysmal supraventricular tachycardia were as follows (measured from the onset of the QRS to the onset of the respective atrial electrogram): V-distal coronary sinus (110 ms), V-high right atrium (145 ms), and V-low septal right atrium (160 ms) (Fig. 1B).

Short periods of functional left and right bundlebranch block were also observed during episodes of induced paroxysmal supraventricular tachycardia. The cycle length during tachycardia with left bundle-branch block was always $25 \mathrm{~ms}$ longer than the cycle length during narrow QRS paroxysmal supraventricular tachycardia and with functional right bundle-branch block. Ventricular extrastimuli $\left(V_{\mathbf{s}}\right)$ were coupled to the $Q R S$ (V) of the tachycardia. At $V-V_{s}$ intervals greater than $345 \mathrm{~ms}$, no reset of the atrial electrogram was noted. At V-V $\mathrm{s}$ intervals between 345 and $290 \mathrm{~ms}$, the corressponding $A$ to $A_{s}$ interval progressively shortened as the $\mathrm{V}-\mathrm{V}_{\mathrm{B}}$ was decreased (Fig. 2). At V-V $\mathrm{B}$ intervals between 345-325 ms, the ventricular extrastimulus $\left(V_{s}\right)$ depolarized the atria $\left(A_{s}\right)$ without depolarizing the $H$ is bundle (Fig. 2A). At V-V $\mathrm{s}$ between 285 to $280 \mathrm{~ms}, \mathrm{~V}_{\mathrm{s}}$ depolarized the atrium and interrupted the tachycardia (Fig. 2C).

The presence of a left-sided concealed extranodal pathway with ability for only retrograde conduction was suggested by the following observations: 1) absence of antegrade pre-excitation on all electrocardiograms and during atrial pacing at multiple sites (Denes et al., 1974). 2) Fixed V-A intervals during incremental and coupled ventricular pacing (Wellens and Durrer, 1974a; Narula, 1974). 3) Abnormal retrograde activation sequence (with distal coronary sinus preceding all other atrial electrograms) during ventricular pacing and during paroxysmal supraventricular tachycardia (Svenson et al., 1974). 4) Lengthening of cycle length of paroxysmal supraventricular tachycardia during functional left bundlebranch block (Coumel and Attuel, 1974; Spurrell et al., 1974a; Neuss et al., 1975). 5) Ability to capture the atria from the ventricles during paroxysmal supraventricular tachycardia without depolarizing the His bundle (Zipes et al., 1974; Neuss et al., 1975). 6) Smooth antegrade AV nodal curves (Wu and Denes, 1975). During paroxysmal supraventricular tachycardia, the left-sided anomalous pathway was used for retrograde conduction and the AV node for antegrade conduction.

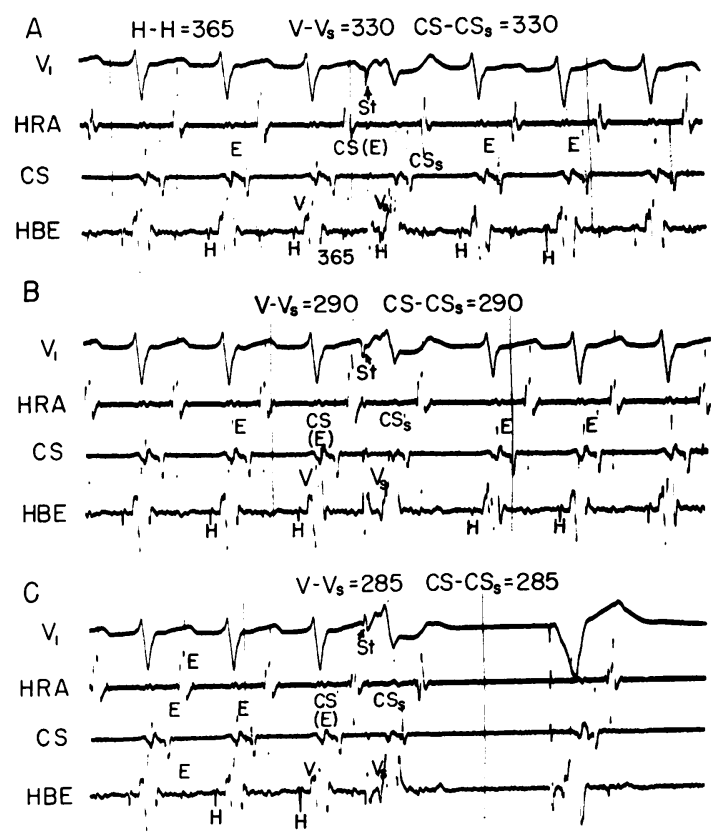

FI G. 2 Atrial reset with premature ventricular stimulation during supraventricular tachycardia. $V$ represents the last $Q R S$ before the introduction of the premature ventricular stimulus $\left(V_{8}\right), C S$ represents the last coronary sinus electrogram before the ventricular extrastimulus. $C S_{8}$ represents the premature coronary sinus electrogram corresponding to the ventricular extrastimulus $\left(V_{8}\right)$. E indicates echo and $H$ represents the His electrogram. The cycle length of the tachycardia is $365 \mathrm{~ms}$. Paper speed is $100 \mathrm{~mm} / \mathrm{s}$, time lines at $1 / s$.

Panel $A$ : The $V-V_{8}$ is 330 ms. The corresponding $C S-C S_{8}$ interval is at $330 \mathrm{~ms}$. Note that the $\mathrm{HH}$ intervals remain undisturbed at $365 \mathrm{~ms}$. This shows that an extranodal pathway must be present. Panel $B$ : The ventricular premature beat is introduced at a $V-V_{8}$ interval of $290 \mathrm{~ms}$. The corresponding $C S-C S_{\delta}$ interval shortens to $290 \mathrm{~ms}$.

Panel C: The $V-V_{s}$ interval is decreased to $285 \mathrm{~ms}$; the corresponding $C S-C S_{8}$ interval is shortened to 285 ms and blocked in the AV node. The tachycardia is then interrupted. The ventricular pacemaker escapes with retrograde conduction to the atria. 


\section{Epicardial mapping and surgery}

Because of medical intractability, and because of the demonstration of a circus movement amenable to correction, the patient was referred to the Texas Heart Institute for elective transection of the His bundle. Operation was performed in March 1975. With the heart exposed, epicardial mapping of the atria was performed during right ventricular pacing using a previously described mapping technique (Svenson et al., 1974; Wyndham et al., 1974). The area of earliest atrial activation was noted at the lateral left atrium just adjacent to the left AV ring (Fig. 3). The patient was placed on cardiopulmonary bypass, and the His bundle was interrupted via a right atriotomy incision. Atrial pacing after closure of the right atriotomy revealed complete AV block. Ventricular pacing after closure of the atriotomy revealed intact retrograde activation. Repeat epicardial atrial mapping with ventricular pacing was identical to that before operation.

\section{Subsequent clinical course}

After operation, the patient recovered uneventfully. He has remained in heart block with pacemaker rhythm and he has been totally free of paroxysmal supraventricular tachycardia. He feels well and has resumed lecturing.

\section{Discussion}

AV re-entry can occur within the AV node or using the normal pathway and an extranodal pathway
(Coumel and Attuel, 1974; Denes et al., 1973; Neuss et al., 1975; Spurrell et al., 1974a, b; Zipes et al., 1974; Wellens and Durrer, 1974a; Svenson et al., 1974; Wu and Denes, 1975). The extranodal pathway can be manifest with ability for antegrade conduction (Wolff-Parkinson-White syndrome) or concealed on the surface electrocardiogram. Concealment of an extranodal pathway reflects unidirectional block with ability for only retrograde conduction. The diagnosis of a concealed extranodal pathway can be suspected by demonstration of the following electrophysiological findings: 1) absence of antegrade pre-excitation on all electrocardiograms and during atrial pacing at multiple sites (Denes et al., 1974). 2) Fixed ventriculoatrial conduction times during incremental and coupled ventricular pacing (Wellens et al., 1974; Narula, 1974). 3) Abnormal sequence of retrograde atrial activation (Svenson et al., 1974). 4) Increase in cycle length of induced paroxysmal supraventricular tachycardia during development of functional bundle-branch block ipsilateral to the suspected anomalous pathway (Coumel and Attuel, 1974; Spurrell et al., 1974a; Neuss et al., 1975). 5) Retrograde capture of the atria during paroxysmal supraventricular tachycardia without discharging the His bundle (Zipes et al., 1974; Neuss et al., 1975). 6) Smooth antegrade conduction curves $\left(\mathbf{A}_{1}-\mathbf{A}_{2}\right.$, $\mathrm{H}_{1}-\mathrm{H}_{2}$ ) with echo zones (Wu and Denes, 1975).

All of the above electrophysiological features were shown in the present case, suggesting the presence

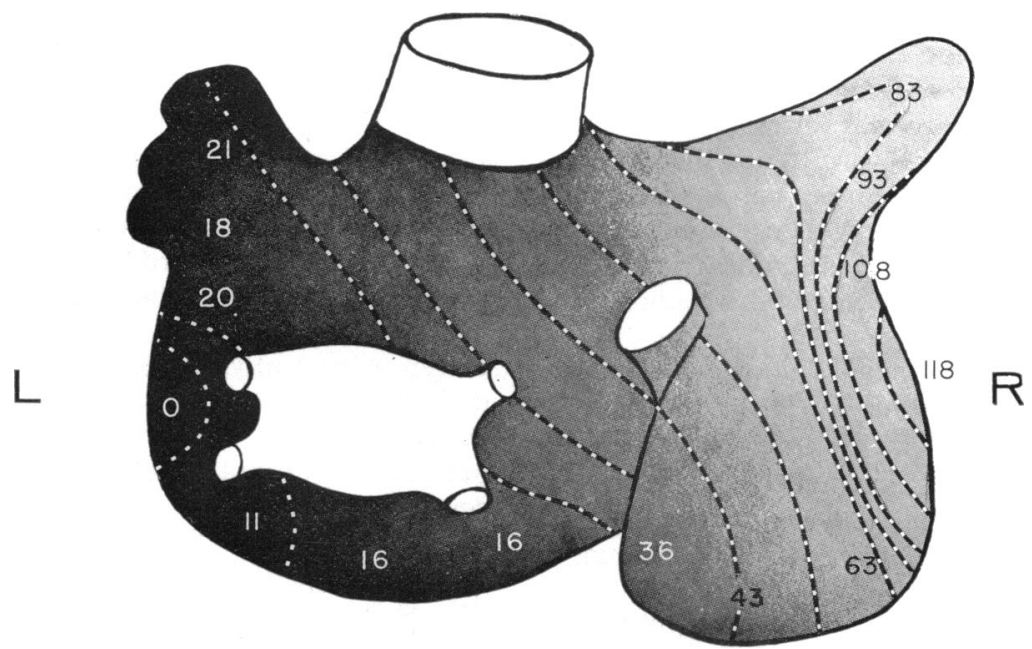

FIG. 3 Posterior schematic view of epicardial atrial mapping during right ventricular stimulation.

Isochronous lines are drawn at 10-ms intervals with reference to the earliest epicardial atrial activation $(0 \mathrm{~ms})$. Note that the posterolateral left atrium contiguous to the left AV ring is activated earliest. 
of a concealed extranodal pathway. In addition, the findings suggested that the extranodal pathway was left-sided. The importance of our case resides in the fact that epicardial mapping of the atria confirmed the presence of a left-sided pathway, as predicted by preoperative electrophysiological studies. Epicardial mapping showed early retrograde activation of the lateral left atrium contiguous to the left AV ring.

Little is known regarding treatment of recurrent supraventricular tachycardia in patients with concealed extranodal pathways. In our experience, these cases are characterized by smooth conduction curves, as opposed to the discontinuous conduction curves often noted in patients with intra AV nodal (dual AV nodal pathway) re-entrant parosyxmal supraventricular tachycardia (Wu and Denes, 1975). Previous studies concerning induction of reentrant tachycardia in the catheterization laboratory before and after drug administration suggest that patients with smooth conduction curves are more likely to have potentiation of induction after drugs which depress AV nodal conduction (ouabain and propranolol) (Wu et al., 1974; Wu et al., 1975). This probably reflects the fact that some of these smooth curve patients have concealed extranodal pathways underlying their paroxysmal supraventricular tachycardia (Wu and Denes, 1975). The administration of a drug depressing the AV node allows a given atrial extrastimulus to provoke greater AV nodal delays after drug administration. This additional delay potentiates the echo phenomenon, since the extranodal pathway is given more time for recovery from antegrade concealed penetration. Drugs such as procainamide and quinidine, which depress antegrade anomalous pathway conduction, should theoretically prolong retrograde refractoriness for the concealed pathway, so that these become incapable of sustaining re-entrant paroxysmal supraventricular tachycardia (Wellens and Durrer, 1974b). However, the present patient was unresponsive to procainamide and quinidine administration.

Both atrial and ventricular pacing have been used with varying degrees of success in the management of patients with intractable paroxysmal supraventricular tachycardia (Kitchen and Goldreyer, 1972). These were unsuccessful in the present case. The failure of ventricular pacing appeared to reflect the very narrow zone of coupling intervals $(5 \mathrm{~ms})$, in which a ventricular extrastimulus could interrupt sustained paroxysmal supraventricular tachycardia. Long periods of random ventricular pacing would be necessary before a random stimulus fell within this critical zone. Programming of a pacemaker to provide a stimulus in this zone could also fail, since it is likely that the zone might well change in timing from day to day.

Before the use of current diagnostic electrophysiological techniques, surgical cure of intractable paroxysmal supraventricular tachycardia had been achieved by destruction of the His bundle (Gianelly et al., 1967). Surgery has also been used successfully in patients with manifest pre-excitation to interrupt re-entrant circus movements (Sealy and Wallace, 1974). Surgical ablation of anomalous pathway after demonstration with epicardial mapping can prevent recurrent circus movement paroxysmal supraventricular tachycardia (and also prevent rapid ventricular rates during paroxysmal atrial fibrillation). Similar cure of circus movement tachycardia has also been achieved in patients with pre-excitation occurring after surgical division of the normal pathway (Dreifus et al., 1968; Wellens et al., 1974).

This is the first reported case of a patient with intractable re-entrant paroxysmal supraventricular tachycardia caused by a proven concealed extranodal pathway cured by surgery. In this case, we chose to interrupt the His bundle, since this was the easiest of the possible procedures for achieving cure of the tachycardia. The fact that the patient was a Jehovah's witness and could not receive blood transfusions was another factor in reaching this decision. This operation had the disadvantage of producing chronic surgical heart block.

The other surgical approach to a case such as the present one would be to ablate surgically the concealed retrograde pathway. Theoretically, this would be ideal, since it would leave the normal conduction system intact without producing permanent heart block. However, technical difficulties might be anticipated in regard to localization of an anomalous pathway when this pathway was only capable of retrograde conduction. We believe that the feasibility of surgery for retrograde concealed pathways needs further exploration.

One additional point of interest relates to the current electrophysiological status of the present patient. He has chronic complete heart block with intact retrograde conduction. He thus resembles previously reported patients with chronic acquired complete heart block and intact retrograde conduction (Castillo and Samet, 1967; Goldreyer and Bigger, 1970). Previous authors have commented on the possibility that these patients have single lesions producing unidirectional heart block. The present case suggests that some patients with heart block and intact retrograde conduction have acquired heart block involving the normal pathway, with a concealed retrograde pathway allowing retrograde conduction. 
We thank Dr. Bernard Kosowsky of St. Elizabeth's Hospital, Boston, for referring the patient, and Drs. Robert Hall and Denton Cooley for allowing us to perform the epicardial mapping at the Texas Heart Institute.

\section{References}

Castillo, C., and Samet, P. (1967). Retrograde conduction in complete heart block. British Heart fournal, 29, 553.

Coumel, P., and Attuel, P. (1974). Reciprocating tachycardia in overt and latent preexcitation. Influence of functional bundle branch block on the rate of the tachycardia. European fournal of Cardiology, 1, 423.

Denes, P., Wu, D., Dhingra, R. C., Chuquimia, R., and Roxen, K. M. (1973). Demonstration of dual A-V nodal pathways in patients with paroxysmal supraventricular tachycardia. Circulation, 48, 549.

Denes, P., Wyndham, C., Dhingra, R., Amat-y-Leon, F., Levitzky, S., and Rosen, K. (1974). Prediction of anomalous pathway location in patients with WPW syndrome using atrial pacing at multiple sites (abstract). Circulation, 50, Suppl. 3, 222.

Dreifus, L. S., Nichols, H., Morse, D., Watanabe, Y., and Truex, R. (1968). Control of recurrent tachycardia of Wolff-Parkinson-White syndrome by surgical ligature of the A-V bundle. Circulation, 38, 1030.

Gianelly, S., Ayres, S. M., Gomprecht, R. F., Conklin, E. F., and Kennedy, R. J. (1967). Therapeutic surgical division of the human conduction system. Fournal of the American Medical Association, 199, 155.

Goldreyer, B. N., and Bigger, J. T. (1970). Ventriculo-atrial conduction in man. Circulation, 41, 935.

Goldreyer, B. N., and Damato, A. N. (1971). The essential role of atrioventricular conduction delay in the initiation of paroxysmal supraventricular tachycardia. Circulation, 43, 679.

Kitchen, J. G., and Goldreyer, B. N. (1972). Demand pacemaker for refractory paroxysmal supraventricular tachycardia. New England fournal of Medicine, 287, 596.

Narula, P. (1974). Retrograde pre-excitation. Comparison of antegrade and retrograde conduction intervals in man. Circulation, 50, 1129.

Neuss, H., Schlepper, M., and Thormann, J. (1975). Analysis of re-entry mechanisms in three patients with concealed Wolff-Parkinson-White syndrome. Circulation, 51, 75.

Scherlag, B., Lau, S. H., Helfant, R. H., Berkowitz, W. D. Stein, E., and Damato, A. N. (1969). Catheter technique for recording His bundle activity in man. Circulation, 39, 13.
Sealy, W. C., and Wallace, A. G. (1974). Surgical treatment of Wolff-Parkinson-White syndrome. Fournal of Thoracic and Cardiovascular Surgerv, 68, 757.

Spurrell, R. A. J., Krikler, D. M., and Sowton, E. (1974a). Retrograde invasion of the bundle branches producing aberration of the QRS complex during supraventricular tachycardia studied by programmed electrical stimulation. Circulation, 50, 487.

Spurrell, R. A. J., Krikler, D. M., and Sowton, E. (1974b). Concealed bypasses of the atrioventricular node in patients with paroxysmal supraventricular tachycardia revealed by intracardiac electrical stimulation and verapamil. American fournal of Cardiology, 33, 590.

Svenson, R. H., Gallagher, J. J., Sealy, W. C., and Wallace, A. G. (1974). An electrophysiologic approach to the surgical treatment of the Wolff-Parkinson-White syndrome. Report of two cases utilizing catheter recording and epicardial mapping techniques. Circulation, 49, 799.

Wellens, H. J., and Durrer, D. (1974a). Patterns of ventriculoatrial conduction in the Wolff-Parkinson-White syndrome. Circulation, 49, 22.

Wellens, H. J. J., and Durrer, D. (1974b). Effect of procaine amide, quinidine and ajamline in the Wolff-ParkinsonWhite syndrome. Circulation, 50, 114.

Wellens, H. J. J., Janse, M. J., Van Dam, R. T., van Capelle, F. J. L., Meijne, N. G., Mellink, H. M., and Durrer, D. (1974). Epicardial mapping and surgical treatment in Wolff-Parkinson-White syndrome type A. American Heart fournal, 88, 69.

Wu, D., and Denes, P. (1975). Mechanisms of paroxysmal supraventricular tachycardia. Archives of Internal Medicine, $135,437$.

Wu, D., Denes, P., Dhingra, R., Khan, A., and Rosen, K. M. (1974). The effects of propranolol on induction of $A-V$ nodal reentrant paroxysmal tachycardia. Circulation, 50, 665.

Wu, D., Wyndham, C., Amat-y-Leon, F., Denes, P., Dhingra, R. C., and Rosen, K. M. (1975). The effects of ouabain on induction of atrioventricular nodal reentrant paroxysmal supraventricular tachycardia. Circulation, 52, 201.

Wyndham, R. C., Amat-y-Leon, F., Denes, P., Dhingra, R. C., Burman, S. O., Pouget, J. M., and Rosen, K. M. (1974). Posterior left ventricular preexcitation: report of a case. Archives of Internal Medicine, 134, 243.

Zipes, D. P., DeJoseph, R. L., and Rothbaum, D. A. (1974). Unusual properties of accessory pathways. Circulation, $49,1200$.

Requests for reprints to Dr. Pablo Denes, University of Illinois Hospital, P.O. Box 6998, Chicago, Illinois 60680, U.S.A. 